\title{
Site-directed spin labelling of proteins by Suzuki-Miyaura coupling via a genetically encoded aryliodide amino acid $\dagger$
}

\author{
Anandi Kugele, (D) $\ddagger^{\mathrm{a}}$ Theresa Sophie Braun, (ID $\ddagger^{\mathrm{a}}$ Pia Widder, (D) ${ }^{a}$ Lara Williams, (ID ${ }^{a}$ \\ Moritz Johannes Schmidt, (DD ${ }^{a}$ Daniel Summerer (D) ${ }^{b}$ and Malte Drescher (D) *a
}

\begin{abstract}
We report site-directed protein spin labelling via Suzuki-Miyaura coupling of a nitroxide boronic acid label with the genetically encoded amino acid 4-iodo-L-phenylalanine. The resulting spin label bears a rigid biphenyl linkage with lower flexibility than spin label R1. It is suitable to obtain defined electron paramagnetic resonance distance distributions and to report protein-membrane interactions and conformational transitions of $\alpha$-synuclein.
\end{abstract}

Site-directed spin labelling (SDSL) in combination with electron paramagnetic resonance (EPR) spectroscopy is an important technique to elucidate the structure and dynamics of proteins. ${ }^{1,2}$ Among available spin labels, nitroxides are especially widely used, ${ }^{3}$ since they can provide insights into the polarity, solvent accessibility and rotational dynamics of the protein sites under study. The observed overall dynamics are thereby composed of tumbling of the whole molecule, flexibility of the secondary structure elements and side chain flexibility of the labelled amino acid itself. ${ }^{4,5}$ These characteristics also enable monitoring interactions of a protein with other biomacromolecules or membranes. ${ }^{6,7}$

The most commonly used nitroxide spin label is the methanethiosulfonate spin label (MTSSL) that readily forms disulfide linkages with accessible cysteines. ${ }^{2}$ However, the requirement of cysteine mutagenesis for site-directed labelling impedes studies of proteins with essential cysteines in their natural state. Moreover, though labelling of surface-exposed membrane proteins has been reported in bacteria, ${ }^{8}$ the high number of sulfhydryl

\footnotetext{
${ }^{a}$ Department of Chemistry and Konstanz Research School Chemical Biology (KoRS-CB), University of Konstanz, Universitätsstraße 10, 78457 Konstanz, Germany. E-mail: malte.drescher@uni-konstanz.de

${ }^{b}$ Faculty of Chemistry and Chemical Biology, TU Dortmund University, Otto-Hahn-Straße 4a, 44227 Dortmund, Germany.

E-mail: daniel.summerer@tu-dortmund.de

$\dagger$ Electronic supplementary information (ESI) available: Experimental details and supplementary figures on the synthesis of NOBA, on expression, isolation and spin labelling of ASYN and TRX, ASYN-vesicle interaction, EPR measurements, spectral simulations, MS, and CD measurements.

\# A. K. and T. S. B. contributed equally.
}

groups in cells and the low redox stability of disulfides complicate in-cell studies.

An alternative are labelling strategies based on noncanonical amino acids (ncAA). This approach relies on the expression of an orthogonal aminoacyl-tRNA-synthetase (aaRS)-tRNA pair capable of selectively charging a nonsense suppressor tRNA with an ncAA, enabling the co-translational incorporation of unique chemical and biophysical functions into proteins in cells at a nonsense codon such as the amber codon. ${ }^{9}$ Stably linked nitroxides can thereby be installed into proteins at defined sites independently of cysteine mutagenesis, either by direct incorporation of nitroxide ncAA or by bioorthogonal conjugation of nitroxide labels to ncAA with unique reactive groups. ${ }^{10}$

While the former approach offers particularly simple labelling directly in $E$. coli and minimal interference with target protein function and host cell, partial loss of paramagnetism of the nitroxide ncAA in E. coli cultures has been reported. ${ }^{11}$

In contrast, conjugations with reactive ncAA ${ }^{12}$ can offer excellent selectivity. However, their potential for nitroxide labelling is underexplored ${ }^{13}$ and they are not always compatible with target protein function or potential in-cell labelling, or result in labels with unfavourable spectroscopic properties. After the use of a ketone-bearing ncAA ${ }^{14}$ for oxime formation, azideand alkyne-bearing ncAA have been reported for click cycloaddition reactions. Biocompatibility has thereby been improved by the use of advanced ligands in copper-catalysed reactions or by exploiting strain-promoted, copper-free chemistries. ${ }^{15-17}$

An emerging strategy for bioorthogonal conjugation is the use of palladium-catalysed coupling reactions. ${ }^{19-21}$ Sonogashira- and Suzuki-Miyaura-type couplings with ncAA have previously been applied to proteins with biosynthetically incorporated ncAA for protein modifications and labelling. ${ }^{22-25}$ Whereas significant improvements of coupling have been reported for palladium catalysts with novel ligands ${ }^{26,27}$ successful ligand-free conditions have also been reported ${ }^{27}$ and both have been applied for cellular labelling. ${ }^{28-30}$ Compared to well-established coppermediated click chemistry, the low toxicity of palladium is advantageous for potential in vivo use. ${ }^{29}$ 
A<smiles>CCCCCCCCCC(C)C</smiles>

\section{B}
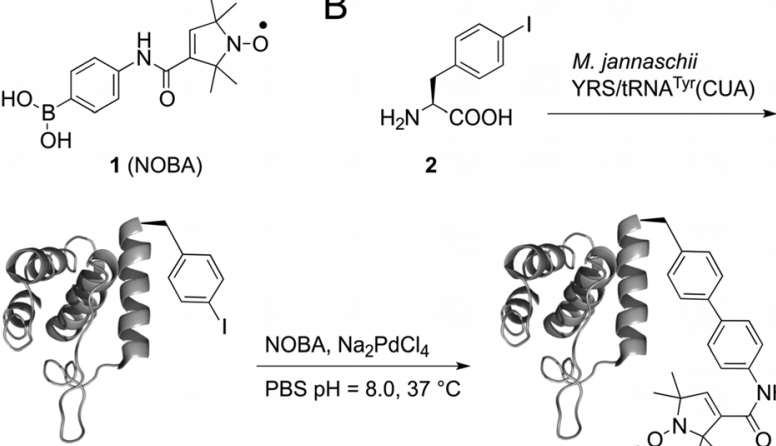

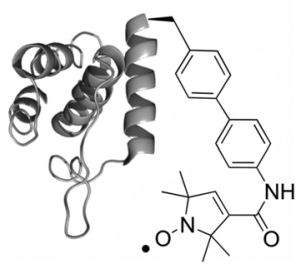

Fig. 1 Site-directed protein spin labelling by Suzuki-Miyaura coupling utilizing the boronic acid spin label NOBA (1) and the genetically encoded ncAA pIPhe (2). (A) Chemical structure of NOBA (1). (B) Incorporation of aryliodide ncAA 2 into proteins and labelling conditions.

We were interested in exploring the applicability of SuzukiMiyaura-type coupling reactions to protein nitroxide labelling. Besides bioorthogonality and the ability to study cysteinecontaining proteins, the formation of stable and highly rigid biphenyl-linkages may offer favourable spectroscopic properties of the resulting conjugate. We designed and synthesized the nitroxide boronic acid label 1 (abbreviated as NOBA, Fig. 1A) by amide formation between 4-aminophenylboronic acid pinacol ester and the respective nitroxide carboxylic acid and subsequent deprotection with polymer-bound boronic acid (see ESI $\dagger$ ).

We next aimed to site-specifically install an aryl iodide for subsequent coupling (Fig. 1B) in E. coli thioredoxin (TRX) because of its important role in redox processes such as dithiole-disulfide exchange reactions. The catalytic center of TRX is constituted of two redox-active cysteines (C33 and C36, Fig. 2A), and X-ray structures ${ }^{18}$ as well as previous EPR studies ${ }^{11,31}$ with different nitroxide labels are available for data comparison. We co-expressed a previously reported Methanocaldococcus jannaschii $\mathrm{tRNA}^{\mathrm{Tyr}} / \mathrm{YRS}$ pair ${ }^{32}$ for the incorporation of 4 -iodoL-phenylalanine 2 (Fig. 1B) with TRX bearing a C-terminal His6 tag and containing an amber codon at position R74 (Fig. 2A). R74 is located in a loop adjacent to the TRX $3_{10}$ helix (Fig. 2A) that exhibited little backbone flexibility in previous molecular dynamics simulations and is suited for the selective assessment of nitroxide label flexibility. ${ }^{31}$ Expressions were conducted in presence of $3 \mathrm{mM} \mathrm{2}$, leading to expression of full-length protein

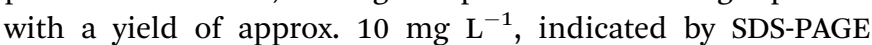
analysis of the protein after purification by Ni-NTA chromatography via the C-terminal His6 tag (see ESI $\dagger$ ). For coupling, we tested palladium catalyst systems with the previously reported ligands dimethyl- and tetramethylguanidine ${ }^{27}$ or with ligandfree $\mathrm{Na}_{2} \mathrm{PdCl}_{4}$, of which only the latter afforded effective labelling under the applied conditions (see ESI $\dagger$ ). Specifically, we incubated purified TRX-R74 $\rightarrow 2$ or aryl iodide-free TRX-R74 (wt, wildtype) with 30 eq. of $\mathrm{Na}_{2} \mathrm{PdCl}_{4}$ as well as 100 eq. of NOBA in PBS buffer, $\mathrm{pH} 8$, at $37^{\circ} \mathrm{C}$ for $3 \mathrm{~h}$. Immediately before removing excess NOBA, DTT was added as a scavenger ${ }^{33}$ to remove undesired palladium adducts (circular dichroism (CD) spectra revealed intact protein conformation after this treatment, see ESI. $\dagger$
A

B
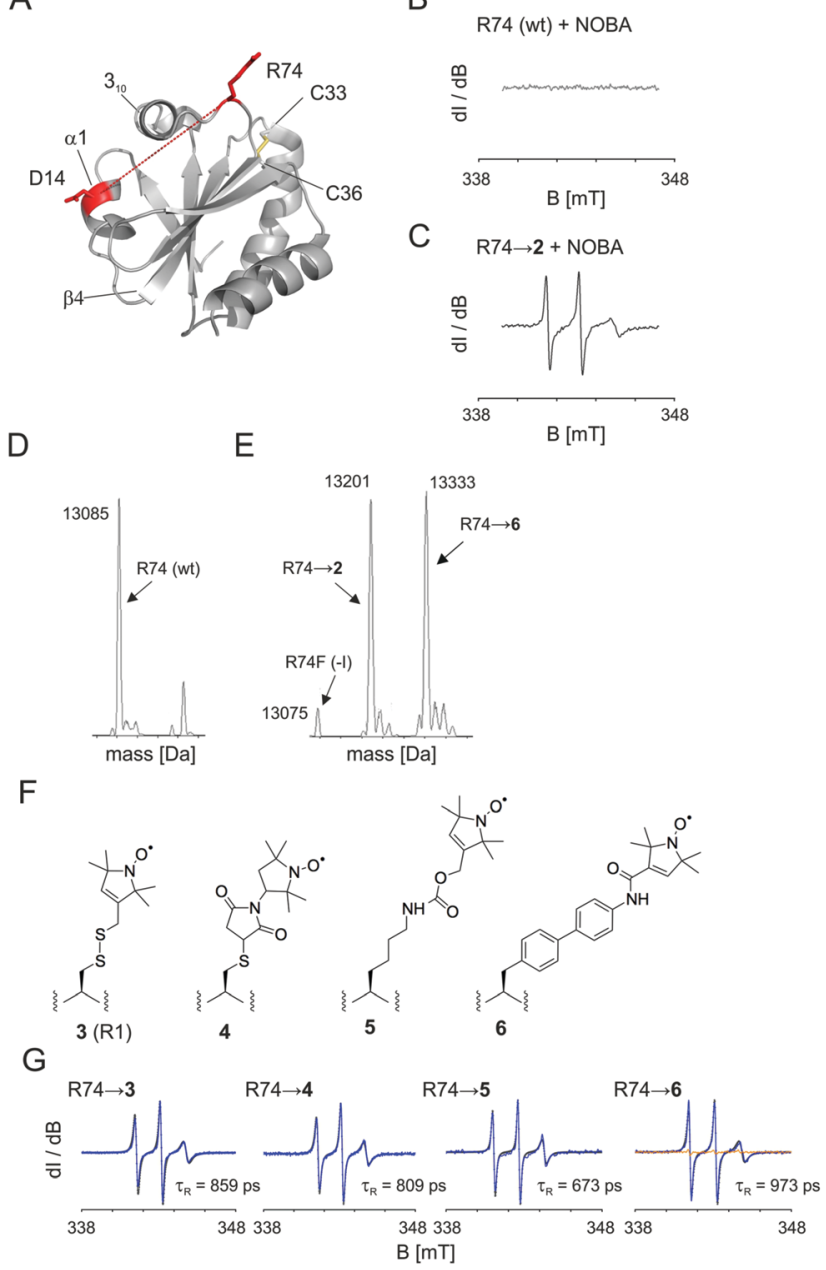

Fig. 2 Site-directed protein spin labelling by Suzuki-Miyaura coupling. (A) Crystal structure of TRX (pdb entry 2TRX). ${ }^{18}$ Incorporation sites of 2 and $\mathrm{C} \alpha-\mathrm{C} \alpha$ distance $(2.1 \AA)$ are shown in red. (B) Cw EPR spectrum of TRX-R74 (wt) reacted with NOBA after purification. (C) Cw EPR spectrum of TRXR74 $\rightarrow 2$ reacted with NOBA after purification. (D) ESI-MS spectrum of sample from (B). (E) ESI-MS spectrum of sample from (C). (F) Structures of spin labelled amino acids used in this study. (G) CW EPR spectra of TRX bearing spin labelled amino acids as indicated at position R74 (blue). The corresponding spectral simulation is shown as dark-grey dotted line. For $\mathrm{R} 74 \rightarrow 6(20 \mu \mathrm{M}$ spin concentration), spectrum of R74 (wt) reacted and purified identically is overlaid in orange $(1 \mu \mathrm{M}$ spin concentration shows minor remaining impurities of unreacted NOBA after purification).

This suggests that labelling of solvent-exposed sites does not interfere with protein integrity. However, it cannot be excluded that labelling of internal sites might interfere with protein stability). Analysis by continuous wave (cw) EPR measurements (Fig. 2B and C) and electrospray ionization mass spectrometry (ESI-MS, Fig. 2D and E) revealed formation of the desired labelling product only for TRX-R74 $\rightarrow 2$, indicating a high chemoselectivity of the reaction. Occurrence of a mass with $\Delta=-126$ Da indicated formation of a minor amount of deiodinated TRX-R74 $\rightarrow 2$ (TRX-R74F), as observed previously for Suzuki-Miyaura reactions (Fig. 2E, see $\mathrm{ESI} \dagger$ for mass interpretation). ${ }^{34}$ Comparative $\mathrm{cw}$ EPR measurements with TRX bearing the standard R1 label $3,^{2}$ the PROXYL-label $4,{ }^{35}$ 
the nitroxide ncAA SLK1 $5^{11}$ or the new NOBA-derived label 6 (Fig. 2F) revealed the highest tumbling time $\tau_{\mathrm{R}}$ of the latter (Fig. 2G), possibly reflecting a comparably low flexibility of the NOBA linker. For further investigation of this aspect, we aimed for EPR distance measurements.

For this purpose, we simultaneously incorporated 2 at positions D14 and R74 in TRX for subsequent labelling and double-electron-electron resonance (DEER) distance measurements (Fig. 2A shows positions). We have previously conducted DEER studies with a TRX C33S/C36S/D14C/R74C mutant doubly labelled with MTSSL (C33S/C36 $\rightarrow$ S/D14/R74 $\rightarrow$ 3), ${ }^{31}$ providing a reference for assessing the spectroscopic properties of 6 . We reacted TRX D14/R74 $\rightarrow 2$ with NOBA as above and subsequently purified and shock-froze the obtained TRX $\mathrm{D} 14 / \mathrm{R} 74 \rightarrow 6$ in deuterated water containing $20 \%$ deuterated glycerol (see ESI $\dagger$ ). Despite the relatively low labelling degree we obtained a DEER trace with a sufficient modulation depth-tonoise ratio (see ESI $\dagger$ ). Similar to the MTSSL-labelled TRX of our previous study ${ }^{31}$ (Fig. 3A), the distance distribution of TRX $\mathrm{D} 14 / \mathrm{R} 74 \rightarrow 6$ showed one main distance between $2-3 \mathrm{~nm}$, as well as two to three less populated higher distances (Fig. 3B, the main distance thereby was somewhat higher for TRX D14/R74 $\rightarrow$ 6). Nuclear magnetic resonance (NMR) studies ${ }^{36}$ and molecular dynamics (MD) simulations ${ }^{31}$ have suggested that the width of TRX-derived distance distributions directly reflects the conformational flexibility of the attached linker. Strikingly, in agreement with the cw measurements (Fig. 2G), the distribution of the main distance was narrower for TRX D14/R74 $\rightarrow 6$ (full width at half maximum $(\mathrm{FWHM})=0.46 \mathrm{~nm}$ ) compared to the MTSSL labelled TRX C33/C36 $\rightarrow$ S/D14/R74 $\rightarrow 3$ (FWHM = $0.95 \mathrm{~nm}$ ), illustrating the rigidity of the biphenyl-based nitroxide side chain (Fig. 3A and B).

Next, we evaluated whether NOBA enables monitoring proteinmembrane interactions. ${ }^{6}$ A model protein well-suited for this purpose is the human intrinsically disordered protein (IDP) $\alpha$-synuclein (ASYN). ${ }^{37}$ ASYN has been associated with Parkinson's disease $^{38}$ and is known for its unusual conformational flexibility. ${ }^{37,39}$ ASYN binds to anionic phospholipid vesicles, resulting in a conformational transition of the N-terminus of ASYN to an $\alpha$-helical conformation (Fig. 4A). ${ }^{40}$ The protein-membrane
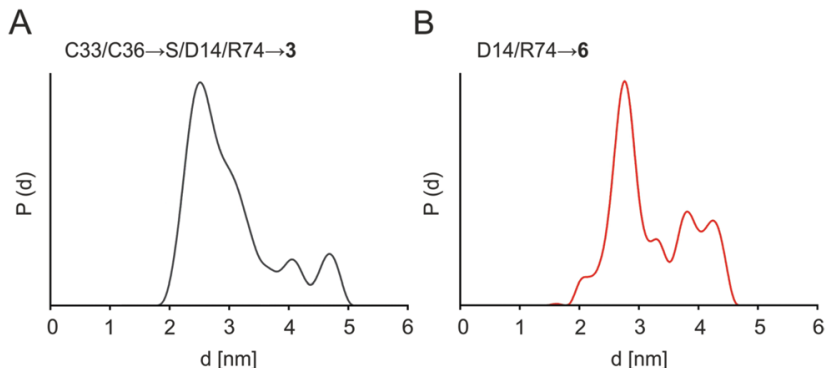

Fig. 3 DEER distance measurements of doubly spin labelled TRX using MTSSL or Suzuki-Miyaura coupling. (A) Distance distribution ${ }^{31}$ for TRX with catalytic cysteines replaced by serine and residues D14 and R74 mutated to cysteine and labelled with MTSSL (C33/C36 $\rightarrow$ S/D14/R74 $\rightarrow$ 3). (B) Distance distribution for TRX D14/R74 $\rightarrow 2$ labelled with NOBA $(\mathrm{D} 14 / \mathrm{R} 74 \rightarrow 6)$.
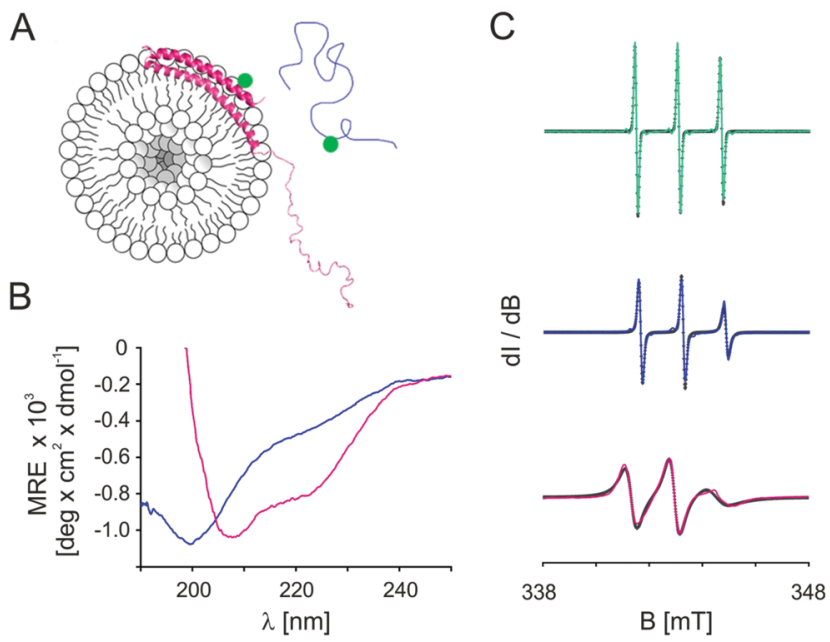

Fig. 4 Spin-labelled ASYN-S9 $\rightarrow 6$ reports interactions with $100 \mathrm{~nm}$ sized POPG vesicles. (A) Schematic representation of disordered ASYN in solution (blue) and of ASYN interacting with a lipid vesicle (magenta, pdb entry $1 X Q 8^{41}$ ). The labelling position S9 used in this study is indicated by a green circle. (B) CD spectra (MRE = molar residue ellipticity) of spinlabelled ASYN-S9 $\rightarrow \mathbf{6}$ in solution (blue) and of spin-labelled ASYN-S9 $\rightarrow \mathbf{6}$ incubated with a 96 -fold excess of POPG LUVs (magenta). The minimum at low wavelengths $(\sim 200 \mathrm{~nm})$ indicates high random coil fractions. Prominent features at 208 and $222 \mathrm{~nm}$ confirm the folding of ASYN into an $\alpha$-helical structure upon vesicle binding. (C) EPR spectra of the unbound spin label NOBA 1 in PBS, pH 8 (green), of spin-labelled ASYN-S9 $\rightarrow \mathbf{6}$ in solution (blue), and of spin-labelled ASYN-S9 $\rightarrow \mathbf{6}$ incubated with a 96 -fold excess of POPG LUVs (magenta). The spectra are normalized to their respective double integral to illustrate spectral differences. The corresponding spectral simulations are shown as dark-grey dotted lines $\left(\tau_{\mathrm{R}}=22 \mathrm{ps}\right.$ for unbound NOBA; $\tau_{\mathrm{R}}=253$ ps for ASYN-S9 $\rightarrow 6$ in solution; $\tau_{\mathrm{R}}=2028$ ps for ASYN-S9 $\rightarrow \mathbf{6}$ bound to vesicles).

interaction has been tracked with local resolution on the residue scale by EPR spectroscopy in combination with conventional MTSSL labels attached to cysteines. ${ }^{6}$ We reacted ASYN-S9 $\rightarrow 2$ with NOBA and purified the protein (see ESI $\dagger$ ). We then incubated the resulting ASYN-S9 $\rightarrow \mathbf{6}$ in the presence or absence of 1-palmitoyl-2-oleoyl-sn-glycero-3-phosphatidylglycerol (POPG) large unilamellar vesicles (LUVs; see ESI $\dagger$ ). In agreement with previous studies, $^{7}$ CD spectra confirmed global protein-membraneinteraction via the spectral indication of the global disorder-to$\alpha$-helical-transition of ASYN upon addition of LUVs (Fig. 4B). Corresponding EPR spectra of ASYN-S9 $\rightarrow 6$ show distinct line broadening upon vesicle addition, indicating reduced rotational mobility, and suggesting membrane binding around residue 9 (Fig. 4C). This shows that the new spin label does not interfere with ASYN-vesicle binding and can be used to monitor proteinmembrane interactions and conformational transitions.

To conclude, we established a chemoselective spin labelling strategy based on Suzuki-Miyaura coupling of a nitroxide boronic acid and a genetically encoded aryliodide amino acid. This allows site-directed protein labelling irrespective of the presence of essential cysteine residues in proteins and under close-to-physiological conditions. The resulting spin labelled amino acid exhibits a stable and rigid biphenyl linkage, and offers attractive spectroscopic properties, as revealed by comparisons 
with previous labels in cw and DEER distance measurements. Labelling of the IDP ASYN and interaction studies with LUVs further reveals that this new label is able to report IDP-membrane interactions and associated conformational transitions. In combination with the previously established biocompatibility of the Suzuki-Miyaura reaction for protein labelling, we anticipate that this label will be useful for a wide range of EPR studies in biological systems.

This project has received funding from the European Research Council (ERC) under the European Union's Horizon 2020 research and innovation programme (Grant Agreement number: 772027 - SPICE - ERC-2017-COG) and from the Deutsche Forschungsgemeinschaft (SFB 969, Project C3). We thank Jessica Dröden, Maria Olivia Schweiss and Artem Fedoseev for experimental contributions and P. G. Schultz for plasmid pEVOL_YRS_pI-Phe.

\section{Conflicts of interest}

There are no conflicts to declare.

\section{Notes and references}

1 O. Griffith, P. Jost and L. Berliner, Spin Labeling: theory and applications, Academic Press, New York, 1976, vol. 1, p. 453.

2 C. Altenbach, T. Marti, H. G. Khorana and W. L. Hubbell, Science, 1990, 248, 1088-1093.

3 W. L. Hubbell, C. J. López, C. Altenbach and Z. Yang, Curr. Opin. Struct. Biol., 2013, 23, 725-733.

4 J. H. Freed, Spin Labeling: theory and applications, Academic Press, New York, 1976, vol. 1, pp. 53-132.

5 W. L. Hubbell, H. S. Mchaourab, C. Altenbach and M. A. Lietzow, Structure, 1996, 4, 779-783.

6 M. Drescher, EPR Spectroscopy, Springer, 2011, pp. 91-119.

7 M. Robotta, J. Cattani, J. C. Martins, V. Subramaniam and M. Drescher, J. Am. Chem. Soc., 2017, 139, 4254-4257.

8 B. Joseph, A. Sikora, E. Bordignon, G. Jeschke, D. S. Cafiso and T. F. Prisner, Angew. Chem., Int. Ed., 2015, 54, 6196-6199.

$9 \mathrm{~J}$. W. Chin, Nature, 2017, 550, 53-60.

10 P. Roser, M. J. Schmidt, M. Drescher and D. Summerer, Org. Biomol. Chem., 2016, 14, 5468-5476.

11 M. J. Schmidt, J. Borbas, M. Drescher and D. Summerer, J. Am. Chem. Soc., 2014, 136, 1238-1241.

12 K. Lang and J. W. Chin, Chem. Rev., 2014, 114, 4764-4806.

13 T. Braun, M. Drescher and D. Summerer, Int. J. Mol. Sci., 2019, 20, 373.
14 M. R. Fleissner, E. M. Brustad, T. Kalai, C. Altenbach, D. Cascio, F. B. Peters, K. Hideg, S. Peuker, P. G. Schultz and W. L. Hubbell, Proc. Natl. Acad. Sci. U. S. A., 2009, 106, 21637.

15 E. H. Abdelkader, A. Feintuch, X. Yao, L. A. Adams, L. Aurelio, B. Graham, D. Goldfarb and G. Otting, Chem. Commun., 2015, 51, 15898-15901.

16 S. Kucher, S. Korneev, S. Tyagi, R. Apfelbaum, D. Grohmann, E. A. Lemke, J. P. Klare, H. J. Steinhoff and D. Klose, J. Magn. Reson., 2017, 275, 38-45.

17 T. Kalai, M. R. Fleissner, J. Jeko, W. L. Hubbell and K. Hideg, Tetrahedron Lett., 2011, 52, 2747-2749.

18 S. K. Katti, D. M. LeMaster and H. Eklund, J. Mol. Biol., 1990, 212, 167.

19 M. Yang, J. Li and P. R. Chen, Chem. Soc. Rev., 2014, 43, 6511-6526.

20 C. P. Ramil and Q. Lin, Chem. Commun., 2013, 49, 11007-11022.

21 N. Miyaura and A. Suzuki, Chem. Rev., 1995, 95, 2457-2483.

22 K. Kodama, S. Fukuzawa, H. Nakayama, T. Kigawa, K. Sakamoto, T. Yabuki, N. Matsuda, M. Shirouzu, K. Takio and K. Tachibana, ChemBioChem, 2006, 7, 134-139.

23 Y. S. Wang, W. K. Russell, Z. Wang, W. Wan, L. E. Dodd, P. J. Pai, D. H. Russell and W. R. Liu, Mol. BioSyst., 2011, 7, 714-717.

24 N. Li, R. K. Lim, S. Edwardraja and Q. Lin, J. Am. Chem. Soc., 2011, 133, 15316-15319.

25 Z. Gao, V. Gouverneur and B. G. Davis, J. Am. Chem. Soc., 2013, 135, 13612-13615.

26 J. M. Chalker, C. S. C. Wood and B. G. Davis, J. Am. Chem. Soc., 2009, 131, 16346-16347.

27 A. Dumas, C. D. Spicer, Z. Gao, T. Takehana, Y. A. Lin, T. Yasukohchi and B. G. Davis, Angew. Chem., 2013, 125, 4008-4013.

$28 \mathrm{~J}$. Li, S. Lin, J. Wang, S. Jia, M. Yang, Z. Hao, X. Zhang and P. R. Chen, J. Am. Chem. Soc., 2013, 135, 7330-7338.

29 C. D. Spicer, T. Triemer and B. G. Davis, J. Am. Chem. Soc., 2011, 134, 800-803.

30 N. Li, C. P. Ramil, R. K. Lim and Q. Lin, ACS Chem. Biol., 2014, 10, 379-384.

31 M. J. Schmidt, A. Fedoseev, D. Bücker, J. Borbas, C. Peter, M. Drescher and D. Summerer, ACS Chem. Biol., 2015, 10, 2764-2771.

32 J. M. Xie, L. Wang, N. Wu, A. Brock, G. Spraggon and P. G. Schultz, Nat. Biotechnol., 2004, 22, 1297-1301.

33 C. D. Spicer and B. G. Davis, Chem. Commun., 2011, 47, 1698-1700.

34 L. Lercher, J. F. McGouran, B. M. Kessler, C. J. Schofield and B. G. Davis, Angew. Chem., Int. Ed., 2013, 52, 10553-10558.

35 O. H. Griffith and H. M. McConnell, Proc. Natl. Acad. Sci. U. S. A., 1966, 55, 8-11.

36 M.-F. Jeng, A. P. Campbell, T. Begley, A. Holmgren, D. A. Case, P. E. Wright and H. J. Dyson, Structure, 1994, 2, 853-868.

37 V. N. Uversky, Front. Aging Neurosci., 2015, 7, 18.

38 J. J. Zarranz, J. Alegre, J. C. Gómez-Esteban, E. Lezcano, R. Ros, I. Ampuero, L. Vidal, J. Hoenicka, O. Rodriguez and B. Atarés, Ann. Neurol., 2004, 55, 164-173.

39 P. H. Weinreb, W. Zhen, A. W. Poon, K. A. Conway and P. T. Lansbury, Biochemistry, 1996, 35, 13709-13715.

40 C. C. Jao, B. G. Hegde, J. Chen, I. S. Haworth and R. Langen, Proc. Natl. Acad. Sci. U. S. A., 2008, 105, 19666-19671.

41 T. S. Ulmer, A. Bax, N. B. Cole and R. L. Nussbaum, J. Biol. Chem., 2005, 280, 9595-9603. 